\title{
Sleep, sex, and the Web: Surveying the difficult-to-reach clinical population suffering from sexsomnia
}

\author{
Michael A. Mangan \\ University of New Hampshire, Durham, New Hampshire \\ AND \\ ULF-DieTRICH REIPS \\ Universität Zürich, Zurich, Switzerland
}

\begin{abstract}
One major advantage of Web-based research lies in its ability to reach and study people who have rare conditions of interest. Another advantage is that, due to the anonymity of the survey situation, the Internet is particularly suited for surveys on sensitive topics. Sexsomnia is a newly identified medical condition whose sufferers engage in sexual behavior during their sleep. Problematic cases are highly distressing and have forensic implications. The consensus among opinion leaders in sleep medicine is that sexsomnia may be quite common but that it often goes unreported because of shame and embarrassment. Thus, little is known about this condition's demographics and clinical features. This article reports findings from a sample analysis of 20 years of research on sexsomnia and discusses the results, strengths, and weaknesses of a recent Web-based survey conducted on the difficult-to-reach clinical population that suffers from sexsomnia.
\end{abstract}

Sexual behavior that occurs during sleep, sexsomnia, is a newly recognized medical condition. It is considered to fall within the broad class of sleep disorders known as parasomnias (Mangan, 2004; Schenck \& Mahowald, 2005; Shapiro, Trajanovic, \& Fedoroff, 2003). Its prevalence is not known. As early as 1986, and very sporadically thereafter, reports describing unusual sexually oriented behavior occurring in sleeping individuals began to appear as case studies in academic journals. Accounts of women's experiences described rape-like situations in which the women's unconscious husbands or boyfriends had violently forced them to have sex. In some instances, these women maintained that their male bed partners were nearly impossible to awaken. In a situation involving sleep masturbation, a woman reportedly had almost nightly episodes of masturbation in her sleep. This put a strain on her relationship, since her male partner was very disturbed by this behavior and did not believe that she was actually asleep (Mangan, 2001, 2004).

Some couples embrace sexsomnia, describing it as an exciting addition to their normal waking sex lives. For example, in one case described by Rosenfeld and Elhajjar (1998), a man had nightly intercourse with his girlfriend but had no recollection of their lovemaking in the morning. One night, he began snoring during intercourse. As comical as this may sound, his snoring was a rather rude awakening for them both to the fact that he was initiating sex in his sleep. This couple eventually sought a medical explanation for his behavior, and they also began to include some of his sleeping sexual behavior into their waking sexual activity.

The handful of published case studies of sexsomnia that existed prior to 1996, along with more recently published research, indicate that sexual behavior in sleep (SBS) covers almost all aspects of human sexual behavior, including fondling, heterosexual and homosexual intercourse, masturbation, and oral sex (i.e., fellatio and cunnilingus). Partners of those acting sexually in their sleep are either willing or unwilling and sometimes include minors under the age of consent. The consensus among sleep medicine practitioners concerning the dangerousness of sexsomnia is that it can pose a threat to relationships, can lead to legal problems for sufferers, and is relatively common but often goes unreported because of shame and embarrassment (Guilleminault, Moscovitch, Yuen, \& Poyares, 2002; Mangan, 2004; Shapiro et al., 2003).

Small numbers of people with sexsomnia typically present only in clinical settings, either because the condition has become troublesome or because they have been referred by a court of law to a sleep clinic for evaluation. Thus, little is known about the demographics of sexsomnia sufferers (e.g., percentages of female and male sufferers) or about sexsomnia's clinical features (e.g., factors that trigger episodes).

M.A. Mangan, mamangan@cisunix.unh.edu 
Sexsomnia is prototypical of cases that are well suited for Internet-based research. Whereas it is very difficult to find and survey a sample of sexsomnia sufferers of sufficient size using traditional surveying methodology, it is relatively easy and convenient to do so using Internetbased research methodology (Reips, 2000, 2002b).

Internet-based research offers a number of advantages over research conducted offline. For example, the 18 advantages listed by Reips (2002b) include the following: ease of access to a large number of demographically and culturally diverse participants as well as to rare and specific participant populations; a reduction of time constraints (research is conducted round the clock); potentially massive parallel participation; scalability (increasing numbers of participants result in almost no increase in effort and cost); and highly voluntary participation that may often help to detect motivational issues via nonresponse rates. Problems of Internet-based research may arise from the researcher's limited control of the situation and from technical issues: Multiple submissions may occur and be difficult to detect; instructions may be misunderstood because no experimenter is present to explain; and technology may interact with sampling (Buchanan \& Reips, 2001).

It is important to keep in mind that all of the benefits and problems associated with computer-based research are also present in Internet-based research. These benefits include automation of data handling, ease of response time measurement, and adaptivity of the procedure (such as questionnaire branching). On the downside, it is difficult to reach people with computer anxiety whereas people with good typing and/or mouse control skills are privileged, and results may not generalize to situations without computers present.

This article reports findings from a sample analysis of 20 years of research on sexsomnia and discusses the results, strengths, and weaknesses of a recent Web-based survey conducted on the difficult-to-reach clinical population that suffers from this condition.

\section{METHOD}

\section{Procedure}

Comparing samples of published studies. Samples from offline and Web-based studies and case reports of SBS published between 1986 and 2006 were reviewed (Alves, Alóe, \& Tavares, 1999; Guilleminault et al., 2002; Hurwitz, Mahowald, \& Schluter, 1989; Rosenfeld \& Elhajjar, 1998; Shapiro, Fedoroff, \& Trajanovic, 1996; Shapiro et al., 2003; Wong, 1986). In the aggregate, 295 participants complained of SBS.

The Web survey. In an attempt to gather descriptive demographic data, a 28-item Web-based survey was constructed, and data were collected using SurveyMonkey, a subscription site at www.surveymonkey.com that supports survey construction, data collection, and analysis. The criterion for inclusion was self-reported personal experience of SBS. No clinical diagnoses were made. Instead, participants' reports of their symptoms were evaluated against clinical knowledge of typical cases.
Links to the survey were sent by e-mail to 409 visitors to Sleepsex.org - a Web site maintained by Michael Mangan - who reported SBS symptoms and provided their e-mail addresses. A link to the study was also placed in a highly visible area of the Sleepsex site. Data were collected over a 3-month period.

A total of 226 responses (157 from the link placed on the site and 69 from the e-mail solicitations) were then carefully evaluated and checked regarding potential challenges in Internet-based data collection (Birnbaum \& Reips, 2005; Reips, 2002a, 2002b).

First, the surveys were screened for responses that did not fit the typical sexsomnia case or were of questionable legitimacy. Seven responses were eliminated because they were clearly bogus (e.g., "I sleep in a barn and had sex with a chicken"). There were five cases of identical Internet protocol (IP) addresses. Each had very different, seemingly valid responses to the survey questions. We determined that the five IPs all belonged to a large Internet provider (AOL) with dynamic IP addressing, meaning that the same IP address can be assigned to different users over the course of time. AOL has more than 30 million users (Holahan, 2006) who share a much smaller pool of IP addresses, so it is inevitable to find several AOL participants with identical IP addresses in a large Web-based study. ${ }^{1}$ With some reservation, we included these 5 respondents' data. It struck us as unlikely that someone would take the time to complete the survey two or three times, giving quite different answers (none of which were bogus) each time (for a discussion, see Reips, 2000). In the end, a total of 219 responses were included.

\section{RESULTS}

\section{Comparing Samples of Published Studies}

In the published studies of sexsomnia, 10\% $(n=30)$ of the participants came from non-Web-based or offline sources (i.e., from persons self-referred to clinics or referred by legal authorities for evaluation as in so-called medicolegal cases), with $90 \%(n=265)$ of participants coming from Web-based sources. The sample sizes in the studies described in the seven articles on sexsomnia published since 1986 that did not use a Web methodology were $1,3,7,2,1,11$, and 5 , in chronological order. ${ }^{2}$ The two Web-based studies that have been conducted (Mangan, 2004; Trajanovic, Mangan, \& Shapiro, 2006) included sample sizes of 46 and 219, respectively (see Figure 1).

\section{The Web Survey}

Strengths. Two of the present Web-based survey's primary strengths were first that it supported access to a population that is often too ashamed or scared to present in a clinical setting and second that it provided easy access to persons with a rare condition (Reips, 2000, 2002b). Also, since sexsomnia sometimes involves adults coming into contact with minors (which was reported by $6 \%$ of the participants in the Web-based study), the legal implications of reporting this to a health care provider are serious. In the United States and Germany, for example, duty-to- 


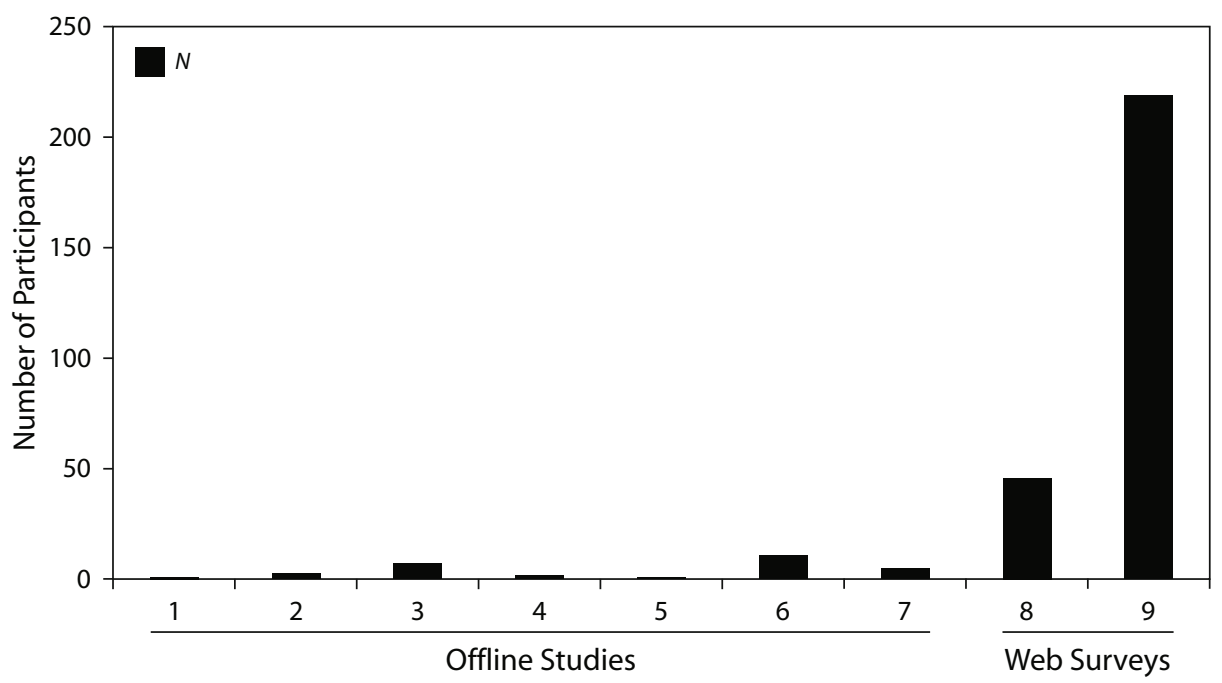

Figure 1. Comparison of sample sizes of published studies.

inform laws require health care providers to report such events to the appropriate authorities. In short, given the increased sense of anonymity that the Web-based survey provided, people probably disclosed facts about their experiences that they might not have revealed in a face-to-face interaction. Another strength of using the Web for research is epistemological advantages associated with the ease of reaching participants across the Internet. The multiple-site entry technique (Reips, 2000, 2002b), for example, allows for easy testing for the presence of self-selection effects.

In the present study, differences between the two data sources across all variables were assessed. The e-mail and Web data were statistically indistinguishable except for the variable gender, for which a chi-square test of independence showed the two variables (gender and source) to be related $\left[\chi^{2}(1, N=219)=4.29, p<.05\right]$. Because the survey responses, when controlled for gender, did not differ according to the recruitment source, we concluded that self-selection may play less of a role than one would expect with the highly sensitive topic addressed by the survey.

The distribution of gender in this Internet-based study among e-mail respondents was $59 \%$ male and $41 \%$ female; among Web-site respondents, it was $74 \%$ male and $26 \%$ female. The ratio of females to males responding by e-mail (.68) exceeded the ratio of females to males responding via the Web (.36). Overall, the distribution of males to females using the Internet method was $69 \%$ male and $31 \%$ female, which shows a greater representation of females as compared with offline studies, in which $77 \%$ of respondents were male and $23 \%$ were female.

Weaknesses. A primary weakness of using a Webbased survey in the present study was that it offered no way to assess respondents for the presence of sexsomnia clinically, which could be done if they presented at a clinic. To include respondents' data in the study, we had to compare their symptoms against symptoms reported in offline samples and, ultimately, take what respondents reported as their symptoms at face value. Also, we would expect to find similarities between offline and Web-based samples on other relevant variables, such as the percentage of respondents reporting a history of other sleep conditions. However, in offline studies, $79 \%$ of participants reported a history of other sleep problems (e.g., parasomnia), whereas in the Internet-based study, $47 \%$ of respondents reported such a history. Although the difference is large enough to be of concern, we suggest, pending further research, that it can be partially explained by the fact that offline studies used clinical samples of persons seeking treatment for sleep disturbances and that clinical interviews provided a much more comprehensive evaluation of the existence of other sleep conditions. Because computer and Web access was necessary to participate, the sample was to some extent self-selected and thus may not generalize to the population of interest.

Due to limitations in the data format provided by SurveyMonkey and the design we used, we were not able to implement some of the techniques for data screening, handling of multiple submissions, or management of dropout recommended by Birnbaum and Reips (2005) and Reips (2002a, 2002b). In particular, information available in the HTTP protocol about a participant's operating system, Web browser, and the referring Web site could have helped to further clarify whether participants with identical IP addresses really accessed the survey from the same computer. If a one-item, one-screen design (Reips, 2002b) had been used, response times could have been analyzed for apparently careless responding (zapping through) and particularly sensitive items; dropout curves could have then been drawn and used for further interpretation.

\section{DISCUSSION}

Web-based methodology can be used effectively to conduct research on rare conditions of interest. The Web survey presented in this article reached more than five times as many people from the target population as had 
been assessed in all previous offline studies combined. The greater percentage of females who participated in the Web-based study suggests that the Web may enable better access to cases of female sexsomnia.

Although the Web provides participants with anonymity that cannot be found in the consulting room of a clinic and is, in this way, ideal for researching sensitive topics, it does present the problem of a lack of a comprehensive clinical evaluation or diagnosis. In short, the validity of the participants' reports must be called into question. However, taking what patients say at face value is routinely done in face-to-face clinical settings as well, unless there is a compelling reason not to do so. Certainly there was no compelling reason to believe that the participants who responded to the direct e-mail solicitation would take the time to seek out information about sexsomnia, express distress, provide their e-mail addresses, and then give bogus answers in response to the survey.

Last, because responding to the survey required access to a computer and the Web, along with a certain amount of motivation to participate, the problem of self-selection threatens the validity of generalizing from the results. However, epidemiological data are often based on the clinical reports of patients who have had the motivation and the means to seek out a health care provider. These reports are also, to some extent, self-selected.

Despite the weaknesses of Web-based research outlined here, we nonetheless conclude that implementing advanced techniques of Web research in this area has already provided further insights into sexsomnia and will continue to do so. The authors are currently conducting two additional Web-based studies that have seen an unprecedented response, due largely to national and international media attention to the article in an October 2006 issue of New Scientist (Marshall, 2006) that reported the preliminary results of the survey discussed here. Although sampling effects will again have to be carefully considered, the wide reach of the current studies will provide further details about the demographics and clinical details of sexsomnia.

\section{AUTHOR NOTE}

Correspondence concerning this article should be addressed to M. A Mangan, Department of Psychology, University of New Hampshire, 10 Library Way, Conant Hall, Durham, NH 03824 (e-mail: mamangan@cisunix .unh.edu) or to U.-D. Reips (e-mail: u.reips@psychologie.unizh.ch).

\section{REFERENCES}

Alves, R., Alóe, F., \& Tavares, S. (1999). Sexual behavior in sleep, sleepwalking and possible REM behavior disorder: A case report. Sleep Research Online, 2, 71-72.
Birnbaum, M. H., \& ReIPS, U.-D. (2005). Behavioral research and data collection via the Internet. In R. W. Proctor \& K.-P. L. Vu (Eds.), The handbook of human factors in Web design (pp. 471-492). Mahwah, NJ: Erlbaum.

Buchanan, T., \& Reips, U.-D. (2001). Platform-dependent biases in online research: Do Mac users really think different? In K. J. Jonas, P. Breuer, B. Schauenburg, \& M. Boos (Eds.), Perspectives on Internet research: Concepts and methods. Retrieved March 6, 2007, from www .psych.uni-goettingen.de/congress/gor-2001/contrib/buchanan-tom.

Guilleminault, C., Moscovitch, A., Yuen, K., \& Poyares, D. (2002). Atypical sexual behavior during sleep. Psychosomatic Medicine, 64, 328-336.

Holahan, C. (2006, July 31). Will less be more for AOL? BusinessWeek. Retrieved March 6, 2007, from www.businessweek.com/technology/ content/jul2006/tc20060731_168094.htm.

Hurwitz, T. D., Mahowald, M. W., \& Schluter, J. L. (1989). Sleeprelated sexual abuse of children [Abstract]. Sleep Research, 18, 246.

Mangan, M. A. (2001). Sleepsex: Uncovered. Philadelphia: Xlibris.

Mangan, M. A. (2004). A phenomenology of problematic sexual behavior occurring in sleep. Archives of Sexual Behavior, 33, 287-293.

Marshall, J. (2006, October 28). Profile of a sexsomniac. New Scientist, $40-43$.

ReIPS, U.-D. (2000). The Web experiment method: Advantages, disadvantages, and solutions. In M. H. Birnbaum (Ed.), Psychological experiments on the Internet (pp. 89-117). San Diego: Academic Press.

REIPS, U.-D. (2002a). Internet-based psychological experimenting: Five dos and five don'ts. Social Science Computer Review, 20, 241-249.

ReIPS, U.-D. (2002b). Standards for Internet-based experimenting. Experimental Psychology, 49, 243-256.

Rosenfeld, D. S., \& ElhajJar, A. J. (1998). Sleepsex: A variant of sleepwalking. Archives of Sexual Behavior, 27, 269-278.

Schenck, C. H., \& Mahowald, M. W. (2005). Rapid eye movement and non-REM sleep parasomnias. Primary Psychiatry, 12, 67-74.

Shapiro, C. M., Fedoroff, J. P., \& Trajanovic, N. N. (1996). Sexual behavior in sleep: A newly described parasomnia [Abstract]. Sleep Research, 25, 367.

Shapiro, C. M., Trajanovic, N. N., \& Fedoroff, J. P. (2003). Sexsomnia: A new parasomnia? Canadian Journal of Psychiatry, 48, 311-317.

Trajanovic, N. N., Mangan, M. A., \& Shapiro, C. M. (2006, June). Sexual behavior in sleep: An Internet survey. Poster presented at the annual meeting of the Associated Professional Sleep Societies, Salt Lake City, UT.

WoNG, K. E. (1986). Masturbation during sleep: A somnambulistic variant? Singapore Medical Journal, 27, 542-543.

\section{NOTES}

1. In fact, the prevalence of sexsomnia may be estimated using figures like these from different providers. There is no reason to believe that sexsomnia would have different prevalence rates among users from different providers.

2. Although 11 cases were reported in Shapiro et al. (2003), 6 of those cases were included in a previous 1996 study and thus are not reported again here.
(Manuscript received December 20, 2006; revision accepted for publication April 10, 2007.) 